\title{
A Cross Sectional Study to Assess the Association of Thyroid Autoantibodies with Thyroid Malignancies in a Tertiary Care Hospital in Bangladesh
}

\author{
Mitan Chakma ${ }^{1}$, Milton Barua ${ }^{2, ~ *, ~ K y a w ~ K h i n ~} \mathbf{U}^{1}$, Mahmud Ullah Faruquee ${ }^{1}$, \\ Sayed Muhammad Alauddin Sharif ${ }^{1}$, Shahin Ibn Rahman ${ }^{3}$, Afjal Hossain ${ }^{4}$, Amanat Ullah ${ }^{5}$ \\ ${ }^{1}$ Department of Otolaryngology, Chittagong Medical College Hospital, Chittagong, Bangladesh \\ ${ }^{2}$ Department of Medicine, Chittagong General Hospital, Chittagong, Bangladesh \\ ${ }^{3}$ Department of Endocrinology, Dhaka National Medical College Hospital, Dhaka, Bangladesh \\ ${ }^{4}$ Department of Endocrinology, US Bangla Medical College, Narayanganj, Bangladesh \\ ${ }^{5}$ Acute Medicine, Medway Maritime Hospital, Kent, UK
}

Email address:

dr.miltonbarua@gmail.com (M. Barua)

${ }^{*}$ Corresponding author

To cite this article:

Mitan Chakma, Milton Barua, Kyaw Khin U, Mahmud Ullah Faruquee, Sayed Muhammad Alauddin Sharif, Shahin Ibn Rahman, Afjal Hossain, Amanat Ullah. A Cross sectional Study to Assess the Association of Thyroid Autoantibodies with Thyroid Malignancies in a Tertiary Care Hospital in Bangladesh. American Journal of Biomedical and Life Sciences. Vol. 9, No. 3, 2021, pp. 166-175.

doi: $10.11648 /$ j.ajbls.20210903.14

Received: April 13, 2021; Accepted: June 15, 2021; Published: June 30, 2021

\begin{abstract}
Thyroid cancer is the most common endocrine malignant lesion and its incidence continues to rise. The aim of this study was to assess association between thyroid antibodies and thyroid malignancies. This was a cross sectional study of 120 patients with thyroid nodules undergoing thyroidectomy with recorded preoperative thyroid antibodies [autoantibodies to thyroglobulin (TgAb) and/or thyroid peroxidase (TPOAb)] levels from 2017 to 2018 admitted at the ENT Department of Chittagong Medical College Hospital, Chittagong. Analysis was done to assess the association between preoperative thyroid antibody levels, fine needle aspiration cytology (FNAC) results, type of thyroid surgery and final histopathology. Mean age of the study population was 35.58 years (SD: \pm 11.36 ). According to the final histopathological diagnosis of the 120 patients $80 \%$ were benign and other $20 \%$ were malignant. Men are more likely to suffer from thyroid malignancy than women (33.3\% versus $18.9 \%)$. Multinodules are predominant group than solitary nodule (55\% versus $45 \%$ ). Though the prevalence of thyroid malignancies is more in solitary group than multinodules group (22.2\% versus $18.2 \%)$ the difference is not statistically significant. Among the benign nodules most of them $(88.5 \%)$ were nodular goiter and among the malignancies most of them were (80.3\%) were papillary thyroid carcinoma. There is moderate agreement between FNA and histopathological diagnosis (kappa is .405). The patients with high TSH level in comparison to low TSH level were significantly associated with thyroid malignancy. The patients with preoperative elevated $\operatorname{TgAb}(\geq 40 \mathrm{IU} / \mathrm{mL})$ has 2.55 times of more chance to have malignant lesion than the patients who has normal $\mathrm{TgAb}$ level. The study revealed that elevated level of $\mathrm{TgAb}$ may act as an independent prediction factor for thyroid malignancy.
\end{abstract}

Keywords: Thyroid Nodule, Autoantibody, Malignancy

\section{Introduction}

Thyroid cancer is a cancer originating from follicular or parafollicular thyroid cells. These cells give rise to both welldifferentiated cancers (i.e., papillary and follicular) and anaplastic thyroid cancer. The second cell type, the $\mathrm{C}$ or parafollicular cell, produces the hormone calcitonin and is the cell of origin for medullary thyroid carcinoma (MTC) [1]. Thyroid cancer is the most common endocrine cancer (approximately $1.0 \%-1.5 \%$ of all new cancers diagnosed each year in the USA) [2], and its incidence has continuously increased in the last three decades all over the world. This trend is present on every continent except Africa [3], where 
detection is possibly insufficient. The increasing incidence is indicated by the annual percent change that in the USA was $2.4 \%$ from 1980 to 1997 and $6.6 \%$ from 1997 to 2009 [4]. Based on recent data, thyroid cancer is the fifth most common cancer in women [5]. Genetic factors, environmental influences, and access to medical care can explain the high variability in the thyroid cancer incidence by geographic area and ethnicity. Recent reports indicated similar age-specific trends by racial/ethnic groups. Although the lowest rates of thyroid cancer are observed in blacks, the greatest rate of papillary thyroid cancer acceleration occurs in black females. Male and female annual percent change was $6.3 \%$ and $7.1 \%$ for white patients, $4.3 \%$ and $8.4 \%$ for black, $4.2 \%$ and $6.7 \%$ for Hispanic and $3.4 \%$ and $6.4 \%$ for Asian patient'srespectively [6].

There was paucity of published reports on the spectrum of thyroid disorders in Bangladesh. The range of thyroid disorders other than iodine deficiencies was considered same in Bangladesh as in other countries of Asia [7]. However the relative prevalence of the different thyroid disorders was dominated by iodine deficiency disorders. Such a study published in 1995 reported $35 \%$ cases of all thyroid disorders to be due to iodine deficiency as the primary etiology. The rest were autoimmune $(26 \%)$, malignant $(2.58 \%)$ and other thyroid disorders [8]. Iodine deficiency is the main cause goiter development in Bangladesh. In Bangladesh, goiter is prevalent in bank of river Jamuna, northern part of the country also hilly areas in Sylhet and Chattogram [9]. Thyroid carcinoma, in most cases, presents, clinically as a solitary nodule or as a dominant nodule within a multinodular thyroid gland. The appearance of a thyroid nodule is a frequent occurrence. In the general population, thyroid nodules are found in $4 \%$ to $7 \%$ of adults through palpation and in $19 \%$ to $67 \%$ through ultrasonography [10]. The challenge to clinicians is to identify the minority of thyroid nodules $(5-15 \%)$ that harbors' malignancy from the majority, which can be managed conservatively [11]. There are a number of well-established predictors of malignancy in thyroid nodules including the finding of hard and fixed lesion on clinical examination, rapid growth of nodules, associated hoarseness, dysphagia or lymphadenopathy, although all of these symptoms and signs are relatively uncommon at diagnosis. Further risk factors include young $(<20$ years) or old age ( $>70$ years), male gender and history of irradiation exposure [11]. Fine needle aspiration (FNA) is currently the most accurate, cost effective method for evaluating thyroid nodules and is the procedure of choice. However, FNA alone may be in sufficient to detect cancer as false negative for malignant cytology occurs in $2-5 \%$ and inadequate cytology occurs in up to $20 \%$ [12]. In addition, many retrospective studies have linked higher serum TSH level with an increased risk of Differentiated thyroid carcinoma (DTC). Although these potential predictors may play an important role in the preoperative diagnosis of thyroid carcinoma, the need for other prediction factors exist [13-15].

As a matter of fact, in an attempt to identify other risk factors to help with risk stratification, serum thyroglobulin
(Tg) and their antibodies have been studied. Previous reports have shown a relationship between elevated measurements of $\mathrm{Tg}$ and well-differentiated thyroidcarcinoma (WDTC). Tg has been recognized as an established tumor marker for thyroid cancer [16-19]. However, serum thyroglobulin antibody ( $\mathrm{TgAb}$ ) levels may interact with $\mathrm{Tg}$ and give a lower serum $\mathrm{Tg}$ value. In fact, $\mathrm{Tg}$ complexed with $\mathrm{TgAb}$ cannot be detected by the currently available immunometric assay methods, which impairs in those cases the clinical utility of $\mathrm{Tg}$ as a prognostic factor for WDTC. However, this has lead to another question on the potential significance of $\mathrm{TgAb}$ in risk stratification of thyroid nodule. Depending on the population studied and the assay used, patients with WDTC have two foldelevated TgAb levels as compared to general population of elevated TgAb level [20]. Also, high titers of $\mathrm{TgAb}$ are present in the serum of most patients with chronic lymphocytic thyroiditis (CLT). In 2010, Kim et al [21]. reported for the first time that a positive $\mathrm{TgAb}$ test was an independent predictor of thyroid nodule malignancy, regardless of the presence of CLT. Subsequently, other reports showed conflicting results. The association between autoimmune thyroiditis (AIT) and thyroid cancer is still not clear despite many previous reports. Anti-thyroid antibodies are good markers for the assessment of thyroid auto immunity. Prevalence of auto immunity in the patients with thyroid disease is quite high in Bangladesh. Moreover, autoimmunity in an appreciable number of thyroid patients may remain undetected unless they are checked for antithyroid antibodies [22]. This led us to conduct this study to assess association between thyroid antibodies and thyroid malignancies. The aim of the present study is to assess whether higher levels of preoperative $\mathrm{TgAb}$ correlate with an increased likelihood of a thyroid nodule being malignant.

\section{Materials and Methods}

This is a hospital based cross sectional study was carried out in the Department of ENT, Chittagong Medical College Hospital Chittagong during the period of July 2017 to June 2018. Consecutive sampling of 120 cases with thyroid nodule who were admitted for thyroidectomy in ENT ward with nclusion criteria include all patients of 18 years and above with thyroid nodule and provided consent for participating in the study but patient with previous thyroid surgery and surgically unfit patients were excluded. Both qualitative and quantitative data were collected by using pre designed case record form. All relevant data that were recorded in a predesigned questionnaire, were processed and analyzed using SPSS Version 23, IBM. Written informed consent was taken from each participant with approval of the study protocol from the Institutional Ethical Committee before commencement of the study. Thyroid profiles, including serum $\mathrm{TgAb}$ and TPOAb levels at fasting state were measured prior to thyrodectomy. The measurements of $\mathrm{TPOAb}$ and $\mathrm{TgAb}$ were performed with an automated analyzer: Siemens Advia Centaur CP immunoassay system/2005/US. The normal ranges for TPOAb and TgAb 
were $0-34 \mathrm{IU} / \mathrm{mL}$ and $0-40 \mathrm{IU} / \mathrm{mL}$, and the analytical sensitivities for TPOAb and $\mathrm{TgAb}$ were $5 \mathrm{IU} / \mathrm{mL}$ and $10 \mathrm{IU} / \mathrm{mL}$, respectively. A titer of greater than the upper limit was defined as positive. Finally after operation, tissue was sent for histopathological diagnosis.

\section{Results}

Table 1. Distribution of the study population by their age and histopathological diagnosis.

\begin{tabular}{|c|c|c|c|c|}
\hline \multirow{2}{*}{ Age, in years } & \multicolumn{2}{|c|}{ Histopathological diagnosis } & \multirow{2}{*}{$\begin{array}{l}\text { Total } \\
(\mathrm{n}=120)\end{array}$} & \multirow{2}{*}{ P value } \\
\hline & Benign $(n=96)$ & Malignant $(n=24)$ & & \\
\hline \multicolumn{5}{|l|}{ Category } \\
\hline$\leq 20$ years & $12(12.5 \%)$ & $1(4.2 \%)$ & $13(10.8 \%)$ & \\
\hline $21-30$ years & $29(30.2 \%)$ & $8(33.3 \%)$ & $37(30.8 \%)$ & $0.58 *$ \\
\hline $31-40$ years & $28(29.2 \%)$ & $9(37.5 \%)$ & $37(30.8 \%)$ & \\
\hline $41-50$ years & $20(20.8 \%)$ & $3(12.5 \%)$ & $23(19.2 \%)$ & \\
\hline$>50$ years & $7(7.3 \%)$ & $3(12.5 \%)$ & $10(8.3 \%)$ & \\
\hline Median (IQR) & $35(27.25-45)$ & $36(28-43.75)$ & $35(28-45)$ & $0.569^{\dagger}$ \\
\hline Mean $( \pm \mathrm{SD})$ & $35.29( \pm 11.96)$ & $36.71( \pm 10.06)$ & $35.58( \pm 11.36)$ & \\
\hline
\end{tabular}

IQR: Intraquartile range; SD: Standard deviation; Data are presented either in frequency (percentge) or in Median (IQR)/Mean ( \pm SD). ${ }^{\dagger}: \mathrm{P}$ value derived from Mann-Whitney U test and not significant; *: P value derived from Fisher's Exact test and not significant.

Table 2. Distribution of the study population by their sex and histopathological diagnosis.

\begin{tabular}{lllll}
\hline \multirow{2}{*}{ Sex } & Histopathological diagnosis & & \multirow{2}{*}{ Total (n=120) } & \multirow{2}{*}{ P value } \\
\cline { 2 - 3 } & Benign $(\mathbf{n}=\mathbf{9 6})$ & Malignant $(\mathbf{n}=\mathbf{2 4})$ & $9(7.5 \%)$ & \\
\hline Male & $6(66.7 \%)$ & $3(33.3 \%)$ & $111(92.5 \%)$ & $0.381 *$ \\
Female & $90(81.1 \%)$ & $21(18.9 \%)$ & & \\
\hline
\end{tabular}

Data are presented either in frequency (percentge); *: P value derived from Fisher's Exact test and not significant.

Table 3. Distribution of the study population by their education and occupation and histopathological diagnosis.

\begin{tabular}{|c|c|c|c|c|}
\hline \multirow{2}{*}{ Variables } & \multicolumn{2}{|c|}{ Histopathological diagnosis } & \multirow{2}{*}{ Total $(n=120)$} & \multirow{2}{*}{ P value } \\
\hline & Benign $(n=96)$ & Malignant $(n=24)$ & & \\
\hline \multicolumn{5}{|l|}{ Education } \\
\hline Up to or below Primary & $81(84.4 \%)$ & $19(79.2 \%)$ & $100(83.3 \%)$ & \multirow{3}{*}{$0.6 .5^{*}$} \\
\hline Up to or below SSC & $12(12.5 \%)$ & $4(16.7 \%)$ & $16(13.3 \%)$ & \\
\hline $\begin{array}{l}\text { Up to HSC or above } \\
\text { Occupation }\end{array}$ & $3(3.1 \%)$ & $1(4.2 \%)$ & $4(3.3 \%)$ & \\
\hline Housewife & $80(33.3 \%)$ & $20(83.3 \%)$ & $100(83.3 \%)$ & \multirow{3}{*}{$0.267^{*}$} \\
\hline Farmer & $5(5.2 \%)$ & $3(12.5 \%)$ & $8(6.7 \%)$ & \\
\hline Others & $11(11.5 \%)$ & $1(4.2 \%)$ & $12(10.0 \%)$ & \\
\hline
\end{tabular}

Data are presented either in frequency (percentge); *: P value derived from Fisher's Exact test and not significant.

Table 4. Distribution of the study population by their risk factors and histopathological diagnosis.

\begin{tabular}{|c|c|c|c|c|}
\hline \multirow{2}{*}{ History of } & \multicolumn{2}{|c|}{ Histopathological diagnosis } & \multirow{2}{*}{ Total $(n=120)$} & \multirow{2}{*}{ Pvalue* } \\
\hline & Benign (n=96) & Malignant (n=24) & & \\
\hline \multicolumn{5}{|c|}{ Family history of thyroid malignancy } \\
\hline Yes & $0(0 \%)$ & $0(0 \%)$ & $0(0 \%)$ & \multirow{3}{*}{--} \\
\hline No & $96(100 \%)$ & $24(100 \%)$ & $120(100 \%)$ & \\
\hline \multicolumn{4}{|c|}{ Radiation exposure } & \\
\hline Yes & $0(0 \%)$ & $0(0 \%)$ & $0(0 \%)$ & \multirow{2}{*}{--} \\
\hline No & $96(100 \%)$ & $24(100 \%)$ & $120(100 \%)$ & \\
\hline
\end{tabular}

Data are presented either in frequency (percentge); *: P value is not calculable.

Table 5. Distribution of the study population by their USG findings and histopathological diagnosis.

\begin{tabular}{|c|c|c|c|c|}
\hline \multirow{2}{*}{ USG findings } & \multicolumn{2}{|c|}{ Histopathological diagnosis } & \multirow{2}{*}{ Total $(n=120)$} & \multirow{2}{*}{ P value } \\
\hline & Benign $(n=96)$ & Malignant $(n=24)$ & & \\
\hline Solitary & $42(77.8 \%)$ & $12(22.2 \%)$ & $54(45 \%)$ & \multirow{2}{*}{$0.582 *$} \\
\hline Multinodular & $54(81.8 \%)$ & $12(18.2 \%)$ & $66(55 \%)$ & \\
\hline
\end{tabular}

Data are presented either in frequency (percentge); *: P value derived from Chi-square test and not significant. 
Table 6. FNAC diagnosis of thyroid nodules.

\begin{tabular}{lll}
\hline Cytologic types of thyroid nodules & $\mathbf{( \% ) W i t h i n ~ g r o u p ~}$ & Percentage of total \\
\hline Benign & & \\
Nodular goiter & $97(91.5 \%)$ & $80.8 \%$ \\
Hashimoto thyroiditis & $5(4.7 \%)$ & $4.2 \%$ \\
Benign neoplasm & $4(3.8 \%)$ & $3.3 \%$ \\
Total & 106 & $88.3 \%$ \\
Malignant & $7(87.5 \%)$ & $5.8 \%$ \\
Papillary carcinoma & $1(12.5 \%)$ & $0.8 \%$ \\
Medullary carcinoma & 8 & $6.7 \%$ \\
Total & & \\
Indeterminate & $6(100 \%)$ & $5.0 \%$ \\
Follicular neoplasia & $6(100 \%)$ & $5.0 \%$ \\
Total & 120 & $100 \%$ \\
Grand total & & \\
\hline
\end{tabular}

Data are presented either in frequency (percentage).

Table 7. Histopathologic diagnosis of thyroid nodules.

\begin{tabular}{lll}
\hline Histopathologic type of thyroid nodules & Within group\% & Percentage of total \\
\hline Benign & & \\
Nodular goiter & $85(88.5 \%)$ & $70.8 \%$ \\
Hashimotos thyroiditis & $5(5.2 \%)$ & $4.2 \%$ \\
Benign neoplasm & $6(6.3 \%)$ & $5.0 \%$ \\
Total & 96 & $80.0 \%$ \\
Malignant & $20(80.3 \%)$ & $16.7 \%$ \\
Papillary carcinoma & $2(8.3 \%)$ & $1.7 \%$ \\
Medullary carcinoma & $2(8.3 \%)$ & $1.7 \%$ \\
Follicular carcinoma & 24 & $20.0 \%$ \\
Total & 120 & $100 \%$ \\
Grand total & & \\
\hline
\end{tabular}

Data are presented either in frequency (percentage).

Table 8. Correlation between cytologic and histopathological diagnosis of the patients.

\begin{tabular}{|c|c|c|c|c|c|c|c|}
\hline \multirow{2}{*}{ Cytologic diagnosis } & \multirow{2}{*}{$\begin{array}{ll}\text { No. } & \text { of } \\
\text { patients }\end{array}$} & \multicolumn{6}{|c|}{ Histologic diagnosis No. of patients } \\
\hline & & NG & HT & BN & PCT & MC & FC \\
\hline Nodular goiter & 97 & 75 & 3 & 6 & 12 & 0 & 1 \\
\hline Hashimotos thyroiditis & 5 & 2 & 2 & 0 & 1 & 0 & 0 \\
\hline Benign neoplasm & 4 & 3 & 0 & 0 & 1 & 0 & 0 \\
\hline Papillary carcinoma & 7 & 1 & 0 & 0 & 6 & 0 & 0 \\
\hline Medullary carcinoma & 1 & 0 & 0 & 0 & 0 & 1 & 0 \\
\hline Follicular neoplasia & 6 & 4 & 0 & 0 & 0 & 1 & 1 \\
\hline
\end{tabular}

Data are presented as frequency. NG: Nodular goiter; HT: Hashimotos thyroiditis; BN: Benign neoplasm; PCT: Papillary thyroid carcinoma; MC: Medullary carcinoma; FC: Follicular carcinoma

Table 9. Correlation between FNAC \& histological diagnosis of thyroid nodules ( $n=114)$.

\begin{tabular}{|c|c|c|c|c|c|c|c|}
\hline \multirow{3}{*}{$\begin{array}{l}\text { Cytological diagnosis } \\
\text { FNAC }\end{array}$} & \multicolumn{5}{|c|}{ Histopathological diagnosis } & \multirow{3}{*}{ Kappa } & \multirow{3}{*}{ P value } \\
\hline & \multirow{2}{*}{ No. of cases } & \multicolumn{2}{|c|}{ Concordant } & \multicolumn{2}{|c|}{ Discordant } & & \\
\hline & & $\mathbf{n}$ & $\%$ & $\mathbf{n}$ & $\%$ & & \\
\hline Benign & 106 & 91 & 85.8 & 15 & 14.2 & \multirow{3}{*}{$0.405^{\mathrm{a}}$} & \multirow{3}{*}{$<0.001 *$} \\
\hline Malignant & 8 & 7 & 87.5 & 1 & 12.5 & & \\
\hline Total & 114 & 98 & 86.65 & 16 & 13.35 & & \\
\hline
\end{tabular}

${ }^{\mathrm{a}}$ Moderate agreement between tests. ${ }^{*} \mathrm{P}$ value derived from Chi-square test and significant.

Table 10. Diagnostic reliability of FNAC of thyroid nodules as compared with histopathological diagnoses ( $n=114)$.

\begin{tabular}{lll}
\hline Statistical parameters & Percentages (\%) & $\mathbf{9 5 \%}$ CI* (\%) \\
\hline Sensitivity & 98.91 & 94.09 to 99.79 \\
Specificity & 31.82 & 13.86 to 54.87 \\
Positive predictive value & 88.85 & 82.0 to 88.91 \\
Negative predictive value & 87.50 & 47.57 to 98.88 \\
Accuracy & 85.96 & 78.21 to 91.76 \\
\hline
\end{tabular}

*CI: Confidence interval 
Table 11. Distribution of the study population by their TgAb level and histopathological diagnosis.

\begin{tabular}{|c|c|c|c|}
\hline \multirow{2}{*}{$\operatorname{Tg} \mathrm{Ab}$, in IU/ml } & \multicolumn{2}{|c|}{ Histopathological diagnosis } & \multirow{2}{*}{ Total $(\mathbf{n}=120)$} \\
\hline & Benign (n=96) & Malignant (n=24) & \\
\hline \multicolumn{4}{|l|}{ Category } \\
\hline Normal $(<40 \mathrm{IU} / \mathrm{ml})$ & $75(84.3 \%)$ & $14(15.7 \%)$ & $89(74.2 \%)$ \\
\hline Elevated $(\geq 40 \mathrm{IU} / \mathrm{ml})$ & $21(67.7 \%)$ & $10(32.2 \%)$ & $31(25.8 \%)$ \\
\hline \multicolumn{4}{|c|}{ Odds ratio: $2.55 ; 95 \%$ CI: (0.992 to 6.562$)$; $\mathrm{p}$ value $=0.048^{\dagger}$} \\
\hline Mean \pm SD & $52 \pm 85.51$ & $99 \pm 158.51$ & $63 \pm 105.07$ \\
\hline Median (IQR) & $23(23-32.77)$ & $25(23-61.05)$ & $23(23-48)$ \\
\hline
\end{tabular}

CI: Confidence interval; IQR: Intraquartile range; SD: Standard deviation; Data are presented either in frequency (percentage) or in Median (IQR)/Mean $( \pm \mathrm{SD}){ }^{\dagger}: \mathrm{P}$ value derived from Chi-square test and is significant.

Table 12. Diagnostic reliability of $T g A b \geq 40 I U / m l$ as a test for thyroid malignancy as compared with histopathological diagnoses ( $n=120)$.

\begin{tabular}{lll}
\hline Statistical parameters & Percentages (\%) & 95\% CI* (\%) \\
\hline Sensitivity & 41.67 & 22.11 to 63.36 \\
Specificity & 78.12 & 68.53 to 85.92 \\
Positive predictive value & 32.26 & 20.62 to 46.60 \\
Negative predictive value & 84.27 & 78.99 to 88.42 \\
Accuracy & 70.83 & 61.84 to 78.77 \\
\hline
\end{tabular}

*CI: Confidence interval

Table 13. Distribution of the study population by their TPOAb level and histopathological diagnosis.

\begin{tabular}{|c|c|c|c|}
\hline \multirow{2}{*}{ TPOAb, in IU/ml } & \multicolumn{2}{|c|}{ Histopathological diagnosis } & \multirow{2}{*}{ Total $(n=120)$} \\
\hline & Benign $(n=96)$ & Malignant $(n=24)$ & \\
\hline \multicolumn{4}{|l|}{ Category } \\
\hline Normal (<35 IU/ml) & $48(78.7 \%)$ & $13(21.3 \%)$ & $61(50.8 \%)$ \\
\hline Elevated ( $\geq 35 \mathrm{IU} / \mathrm{ml})$ & $48(81.4 \%)$ & $11(18.6 \%)$ & $59(49.2 \%)$ \\
\hline \multicolumn{4}{|c|}{ Odds ratio: $0.846 ; 95 \%$ CI: $(0.345$ to 2.075$) ; p$ value $=0.846$} \\
\hline Mean \pm SD & $139.08 \pm 313.73$ & $197.21 \pm 427.29$ & $150 \pm 338.24$ \\
\hline Median (IQR) & $28.50(20-63.5)$ & $20(20-66.73)$ & $20(20-64.4)$ \\
\hline
\end{tabular}

CI: Confidence interval; IQR: Intraquartile range; SD: Standard deviation; Data are presented either in frequency (percentage) or in Median (IQR)/Mean $( \pm \mathrm{SD}){ }^{\dagger}$ : P value derived from Chi-square test and not significant.

Table 14. Distribution of the study population by their TSH level and histopathological diagnosis.

\begin{tabular}{|c|c|c|c|}
\hline \multirow{2}{*}{ TSH, in IU/ml } & \multicolumn{2}{|c|}{ Histopathological diagnosis } & \multirow{2}{*}{ Total $(n=120)$} \\
\hline & Benign (n=96) & Malignant (n=24) & \\
\hline \multicolumn{4}{|l|}{ Category } \\
\hline Low $(<0.4)$ & $22(22.9 \%)$ & $2(8.3 \%)$ & $24(20.0 \%)$ \\
\hline Normal (0.4-4.5) & 69 (71.9\%) & $19(79.2 \%)$ & $88(73.3 \%)$ \\
\hline Elevated $(>4.5)$ & $5(5.2 \%)$ & $3(12.5 \%)$ & $8(6.7 \%)$ \\
\hline \multicolumn{4}{|c|}{$\mathrm{p}$ value $=0.149$ (Derived from Fisher's Exact test $\&$ not significant } \\
\hline Mean \pm SD & $1.52 \pm 3.39$ & $1.69 \pm 1.89$ & $1.56 \pm 3.15$ \\
\hline Median (IQR) & $0.76(0.5-1.33)$ & $1.06(0.5-1.53)$ & $0.84(0.44-1.38)$ \\
\hline
\end{tabular}

IQR: Intraquartile range; SD: Standard deviation; Data are presented either in frequency (percentage) or in Median (IQR)/Mean ( \pm SD).

Table 15. Association between thyroid antibodies and indeterminate FNA types.

\begin{tabular}{lllll}
\hline SI. No. & FNA result & Histoligic type & TgAb level & TPOAb level \\
\hline 1. & Follicular neoplasia & Medullary carcinoma & Elevated & Elevated \\
2 & Follicular neoplasia & Follicular carcinoma & Elevated & Elevated \\
3. & Follicular neoplasia & Nodular goiter & Normal & Elevated \\
4. & Follicular neoplasia & Nodular goiter & Normal & Normal \\
5. & Follicular neoplasia & Nodular goiter & Normal & Normal \\
6. & Follicular neoplasia & Nodular goiter & Normal & Normal \\
\hline
\end{tabular}


Table 16. Univariate association of TgAb with age, sex, nodule type, TSH and TPOAb level of the patients.

\begin{tabular}{|c|c|c|c|}
\hline \multirow{3}{*}{ Variables } & \multicolumn{2}{|c|}{ TgAb level of the patient } & \multirow{3}{*}{ Pvalue } \\
\hline & Normal $(<40 \mathrm{IU} / \mathrm{ml})$ & Elevated $(\geq 40 I \mathrm{IU} / \mathrm{ml})$ & \\
\hline & $(n=89)$ & $(\mathrm{n}=\mathbf{3 1})$ & \\
\hline \multicolumn{4}{|l|}{ Age, in years } \\
\hline Median (IQR) & $35(28-45)$ & $35(26-42)$ & $0.445^{\dagger}$ \\
\hline \multicolumn{4}{|l|}{ Sex } \\
\hline Male $(n=9)$ & $7(77.8 \%)$ & $2(22.2 \%)$ & $0.797 *$ \\
\hline Female $(\mathrm{n}=111)$ & $82(73.9 \%)$ & $29(26.1 \%)$ & \\
\hline \multicolumn{4}{|l|}{ Nodule type } \\
\hline Solitary $(\mathrm{n}=54)$ & $42(77.8 \%)$ & $12(22.2 \%)$ & $0.44 *$ \\
\hline Multinodular $(\mathrm{n}=66)$ & $47(71.2 \%)$ & $19(28.8 \%)$ & \\
\hline \multicolumn{4}{|l|}{ TPOAb level } \\
\hline Normal $(n=61)$ & $55(90.2 \%)$ & $6(9.8 \%)$ & $<0.001^{* *}$ \\
\hline Elevated $(n=59)$ & $34(57.6 \%)$ & $25(42.4 \%)$ & \\
\hline \multicolumn{4}{|l|}{ TSH level } \\
\hline Low $(n=24)$ & $16(18.0 \%)$ & $8(25.8 \%)$ & \\
\hline Normal $(\mathrm{n}=88)$ & $68(76.4 \%)$ & $20(64.5 \%)$ & $0.405^{*}$ \\
\hline Elevated $(n=8)$ & $5(5.6 \%)$ & $3(9.7 \%)$ & \\
\hline
\end{tabular}

IQR: Intraquartile range; Data are presented either in frequency (percentage) or in Median (IQR). ${ }^{\dagger}:$ P value derived from Mann-Whitney $U$ test and not significant; *: P value derived from Fisher's Exact test and not significant.**: P value derived from Fisher's Exact test and significant statistically.

Table 17. Univariate association of TPOAb with age, sex, nodule type, and TSH level of the patients.

\begin{tabular}{|c|c|c|c|}
\hline \multirow{3}{*}{ Variables } & \multicolumn{2}{|c|}{ TPOAb level of the patient } & \multirow{3}{*}{ P value } \\
\hline & Normal $(<35 \mathrm{IU} / \mathrm{ml})$ & Elevated $(\geq 35 \mathrm{IU} / \mathrm{ml})$ & \\
\hline & $(\mathrm{n}=61)$ & $(n=59)$ & \\
\hline \multicolumn{4}{|l|}{ Age, in years } \\
\hline Median (IQR) & 38 928-45.5) & $32(26-40)$ & $0.045^{\dagger}$ \\
\hline \multicolumn{4}{|l|}{ Sex } \\
\hline Male $(n=9)$ & $3(33.3 \%)$ & $6(66.7 \%)$ & $0.275^{*}$ \\
\hline Female $(n=111)$ & $58(52.3 \%)$ & $53(47.7 \%)$ & \\
\hline \multicolumn{4}{|l|}{ Nodule type } \\
\hline Solitary $(\mathrm{n}=54)$ & $27(50.0 \%)$ & $27(50.0 \%)$ & $0.869 *$ \\
\hline Multinodular $(\mathrm{n}=66)$ & $34(51.5 \%)$ & $32(48.5 \%)$ & \\
\hline \multicolumn{4}{|l|}{ TSH level } \\
\hline Low $(n=24)$ & $12(19.7 \%)$ & $12(20.3 \%)$ & \\
\hline Normal $(n=88)$ & $49(80.3 \%)$ & $39(66.1 \%)$ & $0.007^{* *}$ \\
\hline Elevated $(n=8)$ & $0(0 \%)$ & $8(13.6 \%)$ & \\
\hline
\end{tabular}

IQR: Intraquartile range; Data are presented either in frequency (percentage) or in Median (IQR). ${ }^{\dagger}$ : P value derived from Mann-Whitney U test and not significant; *: P value derived from Fisher's Exact test and not significant**: P value derived from Fisher's Exact test and significant statistically.

Table 18. Multivariate logistic regression analysis predicting thyroid malignancies.

\begin{tabular}{lll}
\hline \multirow{2}{*}{ Variables } & Oddsratio(OR) & $P$ value \\
\cline { 2 - 3 } & $\mathbf{( 9 5 \% \text { confidence interval) }}$ & $0.924^{*}$ \\
Age, in years & $1.002(0.960-1.046)$ & $0.261^{*}$ \\
Sex, male & $2.551(0.498-13.079)$ & $0.544^{*}$ \\
Solitary nodule & $1.334(0.526-3.386)$ & $0.019^{* *}$ \\
TgAb $\geq 40 I U / \mathrm{mL}^{\mathrm{a}}$ & $3.724(1.236-11.216)$ & $0.197^{*}$ \\
TPOAb $\geq 35 \mathrm{IU} / \mathrm{mL}^{\mathrm{b}}$ & $0.485(0.162-1.455)$ & $0.109^{*}$ \\
TSH, Low vs. Normal & $3.827(0.741-19.77)$ & $0.027^{* *}$ \\
TSH, Low vs. High & $13.93(1.36-42.87)$ & \\
\hline
\end{tabular}

*: Not significant; **: Significant; Serum TgAb and serum TPOAb concentrations were analyzed as categorical variables. ${ }^{\mathrm{a}} \mathrm{Compared}$ with $\mathrm{TgAb}<40 \mathrm{IU} / \mathrm{mL}$ group. ${ }^{\text {bC } C o m p a r e d ~ w i t h ~ T P O A b ~}<35 \mathrm{IU} / \mathrm{mLgroup}$.

\section{Discussion}

The present study was conducted in ENT Department of Chittagong Medical College Hospital, Chattogram, Bangladesh to assess association between thyroid antibodies ( $\mathrm{TgAb}$ and $\mathrm{TPOAB}$ ) and thyroid malignancies among 120 adults with thyroid nodules who were undergone thyroidectomy between July 2017 to June 2018. As a result, we observed that high levels of $\mathrm{TgAb}$ were associated with increased risk of thyroid malignancy. The current study demonstrates that the prevalence of thyroid malignancy was higher in patients with high $\mathrm{TgAb}$, compared to patients with normal $\mathrm{TgAb}$. Our results suggest that a $\mathrm{TgAb}$ count $\geq 40$ $\mathrm{IU} / \mathrm{ml}$ may be specific for thyroid malignancy, although a lower count should not be used to rule out malignancy. In accordance with our data, other authors have reported that 
elevated TgAb levels could be an indicator that a thyroid nodule is at increased risk for malignancy. As mentioned earlier, Kim et al. were the first to report that a positive $\mathrm{TgAb}$ test was an independent predictor of thyroid nodule malignancy, regardless of the presence of CLT [21]. A more recent study conducted by Grani et al. showed that an isolated $\mathrm{TgAb}$ positivity could be a mild risk factor for thyroid cancer, as opposed to Hashimoto's thyroiditis, which did not correlate positively with malignant pathology [35]. Similarly Hosseini et al., suggested that an elevated TgAb level might indicate that a thyroid nodules is at increased risk for malignancy [30]. Although several studies reported there was no relationship between thyroid autoantibodies and thyroid cancer, they were not focused on $\operatorname{TgAb}$. Boelaert et al., evaluated the relationship by analyzing only TPOAb and not $\mathrm{TgAb}$ [36]. Other studies [37, 38] evaluated the association with malignancy by considering positive thyroid autoantibodies as a whole, including TPOAb and TgAb, but association with individual antibodies were not considered. Further analysis in our study revealed that $\mathrm{TAb} \geq 40 \mathrm{IU} / \mathrm{mL}$ might be a predictive marker for thyroid carcinoma independent of other potentially confounding factors such as age, single nodule, and elevated TSH level. To the best of our knowledge, this is the first large retrospective study examining the relationship between ATAs and thyroid in Bangladeshi population. Similar independent predictive value of TgAb level $\geq 40 \mathrm{IU} / \mathrm{ml}$ for DTC was also observed by Quin et al., in Chinese population [31]. Since Daileyet al. [39] proposed an association between thyroid malignancy and Hashimoto disease as stated by Qin et al., the relationship between these two disorders has long been disputed. In one recent prospective study, [40] Azizi et al. suggested that the association between Hashimoto disease and thyroid cancer is antibody specific. In partial agreement with it, we observed that elevated $\mathrm{TgAb}$ concentration is associated with high prevalence of thyroid malignancy but not with elevated TPOAb. It means that, our study showed that AIT was not predictive of malignancy. It is because TPOAb, a more specific serum marker of AIT, showed no association with malignancy and because histological AIT was not present in malignant specimens. Therefore, our study results could be interpreted that $\mathrm{TgAb}$ itself is a predictor of thyroid cancer, not a reflecting of AIT. This was in full agreement with the retrospective review of Kim et al [21]. Measurement of TgAb was stressed in thyroid cancer, because it could interfere with measurement of Tg levels [41], in thyroid malignancy, the prevalence of $\mathrm{TgAb}, 10-30 \%$, [42] is much higher than that of normal population and decreases subsequently after cancer treatment in 3 years [43]. So, some studies suggest that persistent increases in $\mathrm{TgAb}$ titers could be an early relapse marker as a Tg surrogate $[44,45]$. These findings imply that $\mathrm{TgAb}$ elevation is present before surgery in malignant nodules.

Regarding TSH, its role of prediction for malignancy was also apparent in our study [21,46]. Malignancy rate was increased with elevated TSH levels and the level $>4.5 \mathrm{IU} / \mathrm{ml}$ was as an independent predictor of malignancy in comparison to level $<0.4 \mathrm{IU} / \mathrm{ml}$. The reason why TSH acts as a predictive factor for malignancy is not ascertained, but it has been proposed that TSH might cause tumor growth through the TSH receptor in WDTC as in normal thyroid tissue, which results in occult cancer progression into overt cancer [46].

Prevalence of malignancy in our study was $20 \%$ among 120 thyroid nodule cases. The rate was in accordance with another study conducted in Bangladesh where the prevalence was $18 \%$ [12]. It was slightly higher than other studies conducted in ordinary thyroid nodules [48]. This might have resulted from the fact that our institution is a tertiary referral hospital. The proportion of PTC among thyroid cancer diagnosed in our study was $80.3 \%$. It was lower than $98.5 \%$ noted in other study [21], but in concordant with previous study conducted in Bangladesh [12].

Many clinical risk factors, such as male gender, younger age, large nodule size, and single nodule, have long been recognized as predictors of malignancy in patients with thyroid nodules [49-51]. When we investigated such clinical variables as risk factors for cancer, we found no such difference regarding age, sex and nodule number. Comparatively small sample size of the present study and differences in inclusion criteria might explain this dissimilarity.

The sensitivity, specificity, positive predictive value and negative predictive value of $\mathrm{TgAb}$ as a predictive parameter of thyroid malignancy were $16.04 \%, 90.67 \%, 65.38 \%$ and $49.58 \%$ respectively in the study of Hosseini et al. In the present study the corresponding figures were $41.67 \%$, $90.67 \%, 65.38 \%$ and $49.08 \%$ respectively. These differences might be explained by the different cut-off values used in the studies. In our study it was $\geq 40 \mathrm{IU} / \mathrm{ml}$ and in the previous study it was $\geq 30 \mathrm{IU} / \mathrm{ml}$. It is important to mention that in the current literature there is no defined threshold of what is considered an elevated TgAb titer in WDTC. Most studies published set their cut-off values according to the recommendations of the assay kit provided by the manufacturer, which are calculated for its use in the diagnosis of CLT. When used for that purpose, the cut-offs are set higher as it is believed that higher titers of TgAb are needed to interfere with $\mathrm{Tg}$ levels in patients with CLT compared to WDTC [52]. In the aforementioned studies by Kim et al [21], and Grani et al. [35], the patient's final diagnostic outcome was established following FNAC, with only a minority of diagnoses confirmed by histological follow-up. One limitation of using FNAC as a diagnostic tool is that the cytological features observed in the sample obtained are not necessarily representative of the entire thyroid tissue and cannot consequently offer a definitive diagnosis. An alternative to this problem consists of using the final pathology report obtained after surgical excision, which is the only diagnostically conclusive method. However, the latter also presents limitations, namely the bias caused by the selection of patients with high suspicion of thyroid cancer, and hence requiring thyroidectomy. This study revealed a high sensitivity and an acceptable specificity for the FNAC 
test in diagnosis of different types of neoplasia. In an Iranian study overall, the sensitivity of FNAC diagnosis was found to be $95.2 \%$, specificity was $68.4 \%$, positive predictive value was $83.3 \%$, negative predictive value was $89.6 \%$, and accuracy was $85.14 \%$ [53]. Moreover, some studies have reported higher rates of sensitivity and specificity than we observed in this research. This contradiction is because these studies dealt with a smaller number of cases and a lower percentage of malignancy [54, 55]. In addition, in some studies, some or all of the undetermined or suspicious cases were excluded from statistical computation [56]. However, the problem is that this will lead to a reduction in the number of reported FN and FP cases and thus result in an exaggerated rate of accuracy [57].

\section{Conclusion}

The presence of elevated $\operatorname{TgAb}$ correlated with an increased risk for thyroid malignancy. High serum $\mathrm{TgAb}$ levels may serve as a predictive marker for DTC independent of TSH levels. Patients who have preoperative elevated TgAb level has significantly higher chance to have malignant lesion though there is no significant association with TPOAb level but preoperative elevated TgAb, TPOAb and high TSH level were independent risk predictors for thyroid malignancy. Due to some limitation in sample size, a large randomized control trial hopefully will be helpful to established the association between autonantibody level with thyroid malinganacy in future.

\section{References}

[1] Carling T, Udelsman R. Thyroidcancer. Ann Rev Med 2014; 65 (1): 125-137.

[2] Curado MP, Edwards B, Shinetal HR. Cancerincidence in five continents. IARC Sci Pub2007; 9: 160.

[3] Kilfoy BA, Zheng T, Holford TR. International patterns and trends in thyroid cancer incidence, 1973-2002. Canc Caus Contl 2009; 20 (5): 525-531.

[4] http://seer.cancer.gov/ statfacts/html/thyro.html

[5] Jemal A, Siegel R, Xu J, Ward E. Cancerstatistics, 2010. CA: A Canc J for Clini 2010; 60 (5): 277-300.

[6] Aschebrook KB, Kaplan KL, Chiu BC, Angelos P, Grogan RH. The acceleration in papillary thyroid cancer incidence rates is similar among racial and ethnic groups in the United States. Annals of Surg Onco 2013; 20 (8): 2746-53.

[7] Weigle DS, Hooton TM, Toivola B et al. Frequency of thyroid disease among Southeast Asian primary care patients. J clin Pharm Ther 1996; 21: 29-35.

[8] Alam MN, Haq SA, Ansari MAJ et al. Spectrum of Thyroid Disorders in IPGMR, Dhaka. Bangladesh J Medicine 1995; 6: 53-58.

[9] Al Robbani AM. Study of Thyroid Carcinoma in Solitary thyroid Nodule. Dinajpur Med Col J 2013Jan; 6 (1): 58-63.
[10] Mazzaferri EL. Thyroid cancer in thyroid nodules: finding a needle in the hay stack. The American J of Medi 1992; 93 (4): 359-362.

[11] Boelaert K. The association between serum TSH concentration and thyroid cancer: Endocrine related cancer 2009; 16: 1065-1072.

[12] Hossain MA, Sarkar MZ, Datta UK, Karim MA, et al. Frequency of malignancy in solitary thyroid nodule and multinodular goiter. Bangladesh J Otorhinolaryngology 2014; 20 (2): 55-65.

[13] Shi L, Li Y, Guan H. Usefulness of serum thyrotropin for risk prediction of differentiated thyroid cancers does not apply to microcarcinomas: results of 1870 Chinese patients with thyroid nodules. Endocrine J 2012; 59 (11): 973-980.

[14] McLeod DSA, Watters KF, Carpenter AD, Ladenson PW, et al. Thyrotropin and thyroid cancer diagnosis: a systematic review and dose response meta-analysis. The $\mathrm{J}$ of clini Endocrino and metabolism 2012; 97 (8): 2682-2692.

[15] Fiore E, Vitti P. Serum TSH and risk of papillary thyroid cancer in nodular thyroid disease. J Clini Endocrino and Metabolism 2012; 97 (4): 1134-1145.

[16] Scheffler P, Forest VI, Leboeuf R, Florea AV, Tamilia M, Sands NB, et al. Serum thyroglobulin improves the sensitivity of the McGill thyroid nodule score for well-differentiated thyroid cancer. Thyroid. 2014; 24 (5): 852-7.

[17] Sands NB, Karls S, Rivera J, Tamilia M, Hier MP, Black MJ, Gologan O, Payne RJ. Preoperative serum thyroglobulin as an adjunct to fine-needle aspiration in predicting welldifferentiated thyroid cancer. J Otolaryngol Head Neck Surg 2010; 39: 669-73.

[18] Hocevar M, Auersperg M. Role of serum thyroglobulin in the pre-operative evaluation of follicular thyroid tumours. Eur $\mathrm{J}$ Surg Oncol 1998; 24:553-7.

[19] Lee EK, Chung KW, Min HS, Kim TS, Kim TH, Ryu JS, Jung YS, Kim SK, Lee YJ. Preoperative serum thyroglobulin as a useful predictive marker to differentiate follicular thyroid cancer from benign nodules in indeterminate nodules. $\mathrm{J}$ Korean Med Sci. 2012; 27: 1014-8.

[20] Spencer CA. Clinical review: Clinical utility of thyroglobulin antibody $(\mathrm{TgAb})$ measurements for patients with differentiated thyroid cancers (DTC). J Clin Endocrinol Metab 2011; 96 (12): 3615-27.

[21] Kim ES, Lim DJ, Baek KH, Lee JM, Kim MK, Kwon HS, et al. Thyroglobulin antibody is associated with increased cancer risk in thyroid nodules. Thyroid 2010; 20 (8): 885-91.

[22] Hasanat MA, Rumi MAK, Alam MN, Hasan KN, et al. Status of antithyroid antibodies in Bangladesh: Postgrad Med J 2000; 76: 345-349.

[23] Polyzos SA, Kita M, Avramids. Thyroid nodules- Stepwise diagnosis and management. Homones 2007; 6 (2): 101-119.

[24] Welker MJ. Thyroid nodules. American family/Physician 2003; 67 (3): 559-66.

[25] Yassa L, Cibas ES, Benson CB, Frates MC, Doubilt PM et al. Longterm assessment of a multidisciplinary approach to thyroid nodule diagnostic evaluation. Cancer 2007; 11: 508516. 
[26] Gleeson MC, Browning G, Burton MJ, Clarke R, Hibbert JN, Jones NC, et al. Scott-Brown's Otorhinolaryngology, Head and neck Surgery. $7^{\text {th }}$ ed. London, Edward Arnold publishers Ltd; 2008. P. 2672-73.

[27] Gleeson MC, Browning G, Burton MJ, Clarke R, Hibbert JN, Jones NC, et al. Scott-Brown's Otorhinolaryngology, Head and neck Surgery. $7^{\text {th }}$ ed. London, Edward Arnold publishers Ltd; 2008. P. 343.

[28] https://en.m.wikipedia.org > wiki >Anti-thyroid autoantibodies

[29] Gleeson MC, Browning G, Burton MJ, Clarke R, Hibbert JN, Jones NC, et al. Scott-Brown's Otorhinolaryngology, Head and neck Surgery. $7^{\text {th }}$ ed. London, Edward Arnold publishers Ltd; 2008. P. 356.

[30] Hosseini S, Payne RJ, Zawawi F, Mlynarek A, Hier MP, et al. Can preoperative thyroglobulin antibody levels be used as a marker for well differentiated thyroid cancer? J of Otolaryngology - Head and Neck Surgery, 2016; 45: 31.

[31] Qin J, Zhenqian Y, Haixia G, Liangfeng S, Yongping L, et al. High thyroglobulin antibody levels increase the risk of differentiated thyroid carcinoma. Disease Markers. 2015; Article ID 648670.

[32] Wong SL, Grodski S, Yeung MJ, Serpell JW. Anti-thyroid antibodies as a predictor of thyroid cancer. ANZ J Surg. 2015; 85 (11): 849-53.

[33] Lee SH, Baek JS, Lee JY, Lim JA, Cho SY, et al. Predictive factors of malignancy in thyroid nodules with a cytological diagnosis of follicular neoplasm. Endocr Pathol. 2013; 24 (4): $177-83$.

[34] Jaheen H, Sakr M. Predictors of malignancy in patients with solitary and multiple thyroid nodules. J of Surg. 2016; 12 (3): 105-110.

[35] Grani G, Calvanese A, Carbotta G, D'Alessandri M, Nesca A, Bianchini $\mathrm{M}$, et al. Thyroid autoimmunity and risk of malignancy in thyroid nodules submitted to fine-needle aspiration cytology. Head Neck. 2013; 24 (5): 852-7.

[36] Boelaert K, Horacek J, Holder RL, Watkinson JC, Sheppard MC, Franklyn JA. Sereum thyrotropin concentration as a novel predictor of malignancy in thyroid nodules investigated by fine-needle aspiration. J Clini Endocri Metabolism, 2006; 91: 4295-4301.

[37] Rago T, DiCoscio G, Ugolini C, Scutari M, Basolo F, et al Clinical features of thyroid autoimmunity are associated with thyroiditis on histology and are not predictive of malignancy in 570 patients in indeterminate nodules on cytology who had a thyroidectomy. Clin Endocrinol, 2007; 67: 363-369.

[38] Fiore ERT, Provenzale MA, Scutari M, Ugolini C, Basolo F, et al. Lower level of TSH are associated to a lower risk of papillary thyroid cancer in patients with thyroid nodular diseases: thyroid autonomy may play a protective role. Endocr Relat Cancer. 2009; 16: 1251-1260.

[39] Dailey ME, Lindsay S, Skahen R. Relation of thyroid neoplasms to Hashimoto disease of the thyroid gland. AMA Archives of Surgery, 1995; 70 (2): 291-297.

[40] Azizi G, Keller JM, Lewisetal M. Association of Hashimoto's thyroiditis with thyroid cancer. Endocrine-Related Cancer, 2014; 21 (6): 845-852.
[41] Baloch Z, Carayon P, Conte-Devolx B, Demers LM, Henry JF, et al. Laboratory medicine practice guidelines: laboratory support for the diagnosis and monitoring of thyroid disease. Thyroid 2003; 13: 1-126.

[42] Spencer C, Fatemi S 2013 Thyroglobulin antibody (TgAb) methods - strengths, pitfalls and clinical utility for monitoring TgAb-positive patients with differentiated thyroid cancer. Best Pract Res Clin Endocrinol Metab 2013. DOI: 10.1016.

[43] Chiovato L, Latrofa F, Breverman LE, Pacini F, Capezzone M, et al. Disapperance of humoreal thyroid autoimmunity after complete removal of thyroid antigen. Ann Inter Med, 2003; 139: 346-351.

[44] Chung JK, Park YJ, Kim TY, So Y, Kim SK, et al. Clinical significance of elevated level of serum antithyroglobulin antibody in patients with differentiated thyroid cancer after thyroid ablation. Clinical endrocrinology 2002; 57 (2): 215221.

[45] Kim WG, Yoon JH, Kim WB, Kim TY, Kim ET, et al. Change of serum anti thyroglobulin antibody levels is useful prediction of clinical recurrence in thyroglobulin- negative patients with differentiated thyroid carcinoma. Journal Clinical Metabolism 2008; 93: 4683-4689.

[46] Arun K, Nayak SK, Gowda S. Observational study on association between serum thyroid stimulating hormone and thyroid malignancy. Int Surg J 2017; 4: 2002-6.

[47] Ichikawa Y, Saito E, Abe Y, Homma M, Muraki T, Ito K. Presence of TSH receptor in thyroid neoplasms. J Clin Endocrinol Metab 1976; 42 (2): 395-8.

[48] Frates MC, Benson CB, Doubilet PM. Prevalence and distribution of carcinoma in patients with solitary and multiple thyroid nodules on sonography. $\mathrm{J}$ of Clini Endocri and Metabolism 2006; 91 (9): 3411-3417.

[49] Rago T, Fiore E, Scutarietal M. Male sex, single nodularity, and young age are associated with the risk of finding a papillary thyroid cancer on fine-needle aspiration cytology in a large series of patients with nodular thyroid disease. European JofEndocrino 2010; 162 (4): 763-770.

[50] Raparia K, Min SK, Mody DR, Anton R, Amrikachi M. Clinical outcomes for "suspicious" category in thyroid fineneedle aspiration biopsy: patient's sex and nodule size are possible predictors of malignancy. Archives of Pathology and Laboratory Medicine 2009; 133 (5): 787-790.

[51] Lubitz CC, Faquin WC, Yang J. Clinical and cytological features predictive of malignancy in thyroid follicular neoplasms. Thyroid 2010; 20 (1): 25-31.

[52] Verburg FA, Luster M, Cupini C, Chiovato L, Duntas L, Elisei $\mathrm{R}$, et al. Implications of thyroglobulin antibody positivity in patients with differentiated thyroid cancer: a clinical position statement. Thyroid 2013; 23 (10): 1211-25.

[53] Hajmanoochehri F, Rabiee E. FNAC accuracy in diagnosis of thyroid neoplasms considering all diagnostic categories of the Bethesda reporting system: A single-institute experience. J Cytol 2015 Oct-Dec; 32 (4): 238-243.

[54] Bagga PK, Mahajan NC. Fine needle aspiration cytology of thyroid swellings: How useful and accurate is it? Indian J Cancer 2010; 47: 437-42. 
[55] Handa U, Garg S, Mohan H, Nagarkar N. Role of fine needle aspiration cytology in diagnosis and management of thyroid lesions: A study on 434 patients. Journal Cytol 2008; 25: 13-7.

[56] Wong LQ, Baloch ZW. Analysis of the Bethesda system for reporting thyroid cytopathology and similar precursor thyroid cytopathology reporting schemes. Adv Anat Pathol 2012; 19: 313-9.

[57] Yang J, Schnadig V, Logrono R, Wasserman PG. Fine-needle aspiration of thyroid nodules: A study of 4703 patients with histologic and clinical correlations. Cancer 2007; 111: 306-15. 